at an accelerating rate (as shown by the RLI) for (ii) mammals, birds, and amphibian species used for food and medicine (with 23 to $36 \%$ of such species threatened with extinction) and (iii) birds that are internationally traded (principally for the pet trade; $8 \%$ threatened). Trends are not yet available for plants and other important utilized animal groups. Three other indicators, which lack trend data, show (iv) $21 \%$ of domesticated animal breeds are at risk of extinction (and 9\% are already extinct); (v) languages spoken by fewer than 1000 people ( $22 \%$ of the current 6900 languages) have lost speakers over the past 40 years and are in danger of disappearing within this century (loss of linguistic diversity being a proxy for loss of indigenous biodiversity knowledge); and (vi) more than 100 million poor people live in remote areas within threatened ecoregions and are therefore likely to be particularly dependent upon biodiversity and the ecosystem services it provides.

Indicator development has progressed substantially since the 2010 target was set. However, there are considerable gaps and heterogeneity in geographic, taxonomic, and temporal coverage of existing indicators, with fewer data for developing countries, for nonvertebrates, and from before 1980 and after 2005 (4, 5, 25). Interlinkages between indicators and the degree to which they are representative are incompletely understood. In addition, there are gaps for several key aspects of state, pressures, responses, and especially benefits $(4,5,7,26)$.

Despite these challenges, there are sufficient data on key dimensions of biodiversity to conclude that at the global scale it is highly unlikely that the 2010 target has been met. Neither individual nor aggregated indicators of the state of biodiversity showed significant reductions in their rates of decline, apart from coral reef condition, for which there has been no further deceleration in decline since the mid-1980s. Furthermore, all pressure indicators showed increasing trends, with none significantly decelerating. Some local systemspecific exceptions with positive trends for particular populations, taxa, and habitats (Table 2) suggest that, with political will and adequate resources, biodiversity loss can be reduced or reversed. More generally, individual and aggregated response indicators showed increasing trends, albeit at a decelerating rate (and with little direct information on whether such actions are effective). Overall, efforts to stem biodiversity loss have clearly been inadequate, with a growing mismatch between increasing pressures and slowing responses.

Our results show that, despite a few encouraging achievements, efforts to address the loss of biodiversity need to be substantially strengthened by reversing detrimental policies, fully integrating biodiversity into broad-scale land-use planning, incorporating its economic value adequately into decision making, and sufficiently targeting, funding and implementing policies that tackle biodiversity loss, among other measures. Sustained investment in coherent global biodiversity monitoring and in- dicators is essential to track and improve the effectiveness of these responses.

References and Notes

1. Secretariat of the Convention on Biological Diversity, Handbook of the Convention on Biological Diversity (Earthscan, London, 2003).

2. United Nations, Millennium Development Goals Indicators (http://unstats.un.org/unsd/mdg/Host.aspx? Content=Indicators/Officiallist.htm, 2008).

3. Convention on Biological Diversity, Framework for monitoring implementation of the achievement of the 2010 target and integration of targets into the thematic programmes of work, COP 8 Decision VIII/15 (www.cbd.int/decisions, 2006).

4. Walpole et al., Science 325, 1503 (2009).

5. Further information is available as supporting material on Science Online.

6. H. M. Pereira, H. David Cooper, Trends Ecol. Evol. 21, 123 (2006).

7. G. M. Mace, ]. E. M. Baillie, Conserv. Biol. 21, 1406 (2007).

8. J. D. Sachs et al., Science 325, 1502 (2009).

9. N. Gilbert, Nature 462, 263 (2009).

10. H. Mooney, G. Mace, Science 325, 1474 (2009).

11. L. Soldaat, H. Visser, M. van Roomen, A. van Strien, J. Ornithol. 148, (S2), 351 (2007).

12. R. M. Fewster, S. T. Buckland, G. M. Siriwardena, S. R. Baillie, J. D. Wilson, Ecology 81, 1970 (2000).

13. B. Collen et al., Conserv. Biol. 23, 317 (2009).

14. Food and Agriculture Organization, Global Forest Resources Assessment 2005 (FAO, Rome, 2006).

15. M. C. Hansen et al., Proc. Natl. Acad. Sci. U.S.A. 105, 9439 (2008).

16. S. H. M. Butchart et al., PLOS ONE 2, e140 (2007).

17. J. C. Vié, C. Hilton-Taylor, S. N. Stuart, Eds., Wildlife in a Changing World (IUCN, Gland, Switzerland, 2008).

18. R. D. Gregory et al., PLOS ONE 4, e4678 (2009)

19. M. C. Ribeiro, J. P. Metzger, A. C. Martensen, F. Ponzoni, M. Hirota, Biol. Conserv. 142, 1141 (2009).

20. C. Nilsson, C. A. Reidy, M. Dynesius, C. Revenga, Science 308, 405 (2005)

21. G. Eken et al., Bioscience 54, 1110 (2004)

22. T. H. Ricketts et al., Proc. Natl. Acad. Sci. U.S.A. 102, 18497 (2005).
23. M. A. McGeoch et al., Divers. Distrib. 16, 95 (2010).

24. SCBD, The Convention on Biological Diversity Plant Conservation Reports (SCBD, Montreal, 2009)

25. B. Collen, M. Ram, T. Zamin, L. McRae, Trop. Conserv. Sci. 1, 75 (2008).

26. A. Balmford, P. Crane, A. Dobson, R. E. Green, G. M. Mace, Philos. Trans. R. Soc. London Ser. B 360, 221 (2005)

27. P. F. Donald et al., Science 317, 810 (2007)

28. S. H. M. Butchart, A. ]. Stattersfield, N. ]. Collar, Oryx 40, 266 (2006)

29. We are grateful for comments, data, or help from R. Akçakaya, L. Alvarez-Filip, A. Angulo, L. Bennun, L. Coad, N. Cox, M. Dubé, C. Estreguil, M. Evans, B. Galil, V. Gaveau, F. Gherardi, S. Goldfinger, R. Green, A. Grigg, P. Herkenrath, C. Hilton-Taylor, M. Hoffmann, E. Kleynhans, J. Lamoreux, S. Livingstone, E. Marais, P. Martin, I. May, A. Milam, K. Noonan-Mooney, H. Pavese, B. Polidoro, C. Pollock, D. Pritchard, J. Schipper, F. Schutyser, V. Shutte, S. Simons, ]. Škorpilová, A. Stattersfield, P. Voňššk, R. Wright, M. Wackernagel, and $M$. Waycott. We acknowledge support from the Global Environment Facility to the 2010 Biodiversity Indicators Partnership; Shell Foundation; European Commission; the Sea Around Us Project (University of British Columbia/Pew Environment Group) to D.P. and R.W.; World Wildlife Fund, The Nature Conservancy, and the University of Queensland to M.H. and F.L.; T. Haas and the New Hampshire Charitable Foundation to K.E.C.; and the National Science Foundation (NSF) to ].-F.L. Opinions and findings expressed here do not necessarily reflect the views of the NSF or other funding bodies.

Supporting Online Material

www.sciencemag.org/cgi/content/full/science.1187512/DC1 Methods

SOM Text

Figs. S1 and S2

Tables S1 to S4

References

Data File 1

26 January 2010; accepted 8 April 2010

Published online 29 April 2010;

$10.1126 /$ science. 1187512

Include this information when citing this paper.

\title{
Plectasin, a Fungal Defensin, Targets the Bacterial Cell Wall Precursor Lipid II
}

Tanja Schneider, ${ }^{1}$ Thomas Kruse, ${ }^{2}$ Reinhard Wimmer, ${ }^{3}$ Imke Wiedemann, ${ }^{1}$ Vera Sass, ${ }^{1}$ Ulrike Pag, ${ }^{1}$ Andrea Jansen, ${ }^{1}$ Allan K. Nielsen, ${ }^{4}$ Per H. Mygind, ${ }^{4}$ Dorotea S. Raventós, ${ }^{4}$ Søren Neve, ${ }^{4}$ Birthe Ravn, ${ }^{4}$ Alexandre M. J. ]. Bonvin, ${ }^{5}$ Leonardo De Maria, ${ }^{4}$ Anders S. Andersen, ${ }^{2,4}$ Lora K. Gammelgaard, ${ }^{4}$ Hans-Georg Sahl, ${ }^{1}$ Hans-Henrik Kristensen ${ }^{4 *}$

Host defense peptides such as defensins are components of innate immunity and have retained antibiotic activity throughout evolution. Their activity is thought to be due to amphipathic structures, which enable binding and disruption of microbial cytoplasmic membranes. Contrary to this, we show that plectasin, a fungal defensin, acts by directly binding the bacterial cell-wall precursor Lipid II. A wide range of genetic and biochemical approaches identify cell-wall biosynthesis as the pathway targeted by plectasin. In vitro assays for cell-wall synthesis identified Lipid II as the specific cellular target. Consistently, binding studies confirmed the formation of an equimolar stoichiometric complex between Lipid II and plectasin. Furthermore, key residues in plectasin involved in complex formation were identified using nuclear magnetic resonance spectroscopy and computational modeling.

$\mathrm{P}$ lectasin is a 40-amino acid residue fungal defensin produced by the saprophytic ascomycete Pseudoplectania nigrella (1).
Plectasin shares primary structural features with defensins from spiders, scorpions, dragonflies and mussels and folds into a cystine-stabilized alpha- 
beta structure $(\mathrm{CS} \alpha \beta)$. In vitro and in animal models of infection, plectasin is potently active against drug-resistant Gram-positive bacteria such as streptococci, whereas the antibacterial spectrum of an improved derivative, NZ2114 (2), also includes staphylococci such as methicillin-resistant Staphylococcus aureus (MRSA).

We set out to determine the molecular target and specific mechanism by which plectasin kills

${ }^{1}$ Pharmaceutical Microbiology Section, Institute for Medical Microbiology, Immunology, and Parasitology, University of Bonn, D-53115 Bonn, Germany. ${ }^{2}$ Statens Serum Institut, 2300 Copenhagen S, Denmark. ${ }^{3}$ Department of Biotechnology, Chemistry, and Environmental Engineering, Aalborg University, DK-9000 Aalborg, Denmark. ${ }^{4}$ Novozymes AS, DK-2880 Bagsvaerd, Denmark. ${ }^{5}$ Department of Chemistry, Faculty of Science, Utrecht University, 3584 CH Utrecht, Netherlands.

*To whom correspondence should be addressed. E-mail: hahk@novozymes.com bacteria. Although many host defense peptides (HDPs) act on and disintegrate the bacterial membrane, several observations suggested that this is not the case for plectasin.

Growth kinetic measurements of the Grampositive bacterium Bacillus subtilis exposed to plectasin clearly demonstrated that plectasin exhibited kinetic behavior similar to cell wallinterfering agents (such as vancomycin, penicillin, and bacitracin) and not to the rapidly lytic membrane-active agents (such as polymyxin and novispirin) or non-lytic antibiotics with replication (ciprofloxacin), transcription (rifampicin), or protein translation (kanamycin, tetracycline) as their primary target (Fig. 1A) (3). Consistently with this, killing kinetics indicated that over a period of approximately one generation time $(0.5$ hours) treated cells were unable to multiply, but remained viable (Fig. 1B inset), before the number of colony-forming units decreased (Fig. 1B).
Next, the effect of plectasin on macromolecular biosynthesis pathways was investigated. The incorporation of radiolabeled isoleucine into protein and of thymidine into nucleic acids was not affected, whereas glucosamine - an essential precursor of bacterial peptidoglycan - was no longer incorporated (Fig. 1C). Lastly, treatment of B. subtilis with plectasin induced severe cell-shape deformations as visualized through phase-contrast microscopy (fig. S1). These characteristics are all typical for compounds interfering with cell-wall biosynthesis rather than for membrane disintegration $(4,5)$. Consistently, neither pore formation as measured by $\mathrm{K}^{+}$efflux (Fig. 1E), nor changes in membrane potential by use of $\mathrm{TPP}^{+}$or $\mathrm{DiBAC}_{4}$ (fig. S2, A and B), nor carboxy-fluorescein efflux from liposomes were detected (fig. S2C). Thus, despite its amphipathic nature, plectasin does not compromise membrane integrity, reducing the risk of unspecific toxicity.
A

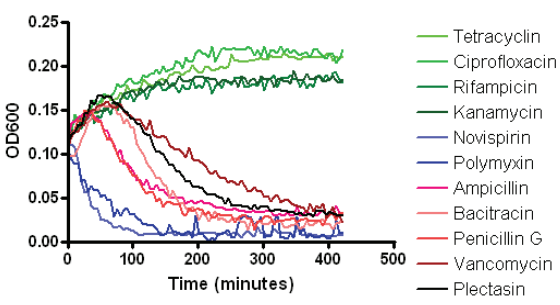

D

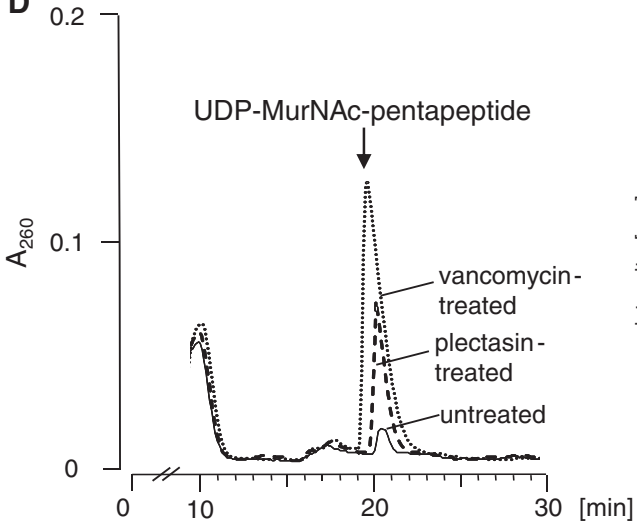

Fig. 1. Effect of plectasin on intact cells. (A) Classification of antimicrobial compounds by using optical density measurements. Growth kinetic measurements of $B$. subtilis exposed to plectasin or various antibiotics with known cellular targets. Two to four times the minimal inhibitory concentration (MIC) of the respective compounds were used. Plectasin (black) falls into the cluster of cell-wall biosynthesis inhibiting antibiotics (red colors). (B) Killing kinetics of plectasin; Staphylococcus simulans 22 treated with plectasin at $2 \times$ MIC (open diamonds) and $4 \times$ MIC (squares); control is without peptide (triangles). (Insert) A similar experiment with more time points within the first 60 min demonstrating the absence of killing in the first 30 min of treatment. (C) Impact of plectasin on macromolecular biosynthesis in B. subtilis 168. Incorporation of $\left[{ }^{14} \mathrm{C}\right]$-thymidine into nucleic acids, of L- $\left[{ }^{14} \mathrm{C}\right]$-isoleucine into protein, and of $\left[{ }^{3} \mathrm{H}\right]$-glucosamine in cell wall was measured in untreated controls (squares) and plectasin-treated cells (open circles); glucosamine incorporation into cell-wall material was selectively inhibited. (D) Intracellular accumulation of the ultimate soluble cell-wall precursor UDP-MurNAc-
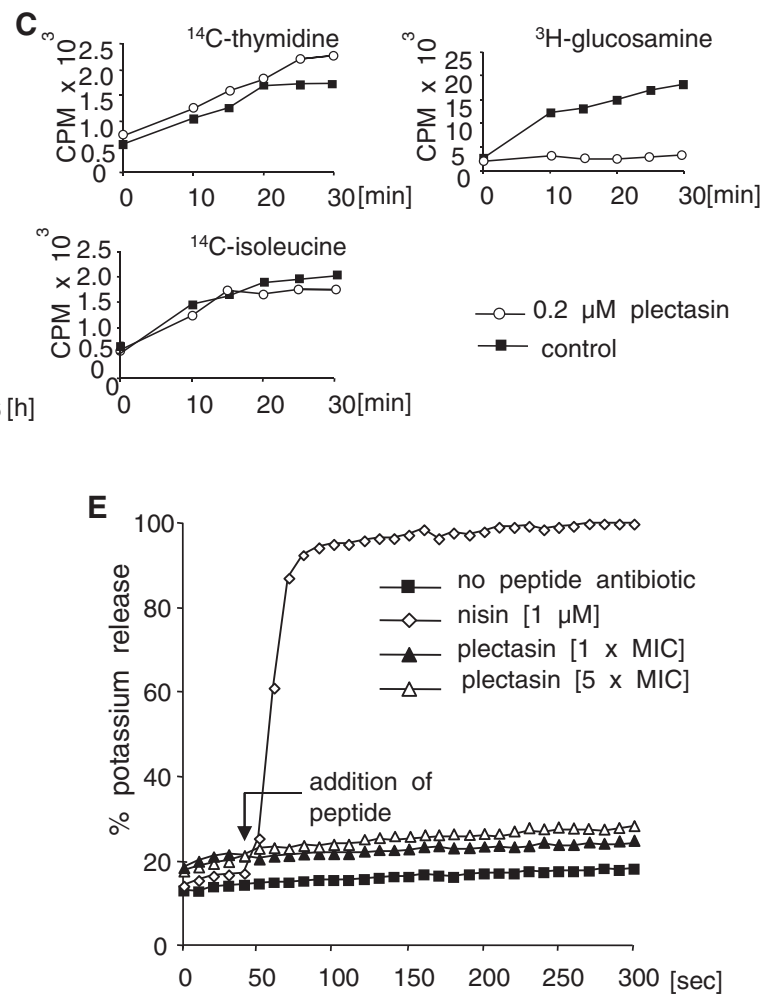

pentapeptide in vancomycin-treated (dotted line) and plectasin-treated (dashed line) cells of $S$. simulans 22. Cells were treated for 30 min with plectasin or vancomycin, which is known to form a complex with Lipid II. Treated cells were extracted with boiling water, and the intracellular nucleotide pool was analyzed by means of reverse HPLC. UDP-MurNAcpentapeptide was identified by means of mass spectrometry using the negative mode and $1 \mathrm{mg} / \mathrm{ml}$ 6-aza-2-thiothymine [in 50\% (v/v) ethanol/20 $\mathrm{mM}$ ammonium citrate] as matrix; the calculated monoisotopic mass is 1149.35; in addition to the singly charged ion, the mono- and disodium salts are detected. (E) Plectasin is unable to form pores in the cytoplasmic membrane of $S$. simulans 22. Potassium efflux from living cells was monitored with a potassium-sensitive electrode. Ion leakage is expressed relative to the total amount of potassium released after addition of $1 \mu \mathrm{M}$ pore-forming lantibiotic nisin (100\%, open diamonds). Plectasin was added at $0.2 \mu \mathrm{M}$ (triangles) and $1 \mu \mathrm{M}$ (open triangles); controls were without peptide antibiotics (squares). 
We obtained further support for the cell wallinterfering activity using DNA microarrays to compare the transcriptional responses of plectasin-treated cells with response patterns obtained for a range of reference antibiotics. For both B. subtilis 168 and $S$. aureus SG511, we found that the transcriptional profiles overlapped those of established cell-wall biosynthesis inhibitors, such as vancomycin and bacitracin (6-9) (fig. S3 and tables S1 and S2).

The biosynthesis of bacterial cell walls requires a number of steps (10). Initially, the $\mathrm{N}$-acetylmuramic acid-pentapeptide (MurNAc-pentapeptide) - a major constituent of the cell-wall building blockis produced in the cytoplasm as an uridine diphosphate (UDP)-activated precursor before it is transferred onto a membrane carrier, bactoprenolphosphate (Fig. 2B, reaction I). The resulting membrane-anchored precursor Lipid I is then fur- ther modified to the structural cell-wall subunit, Lipid II (Fig. 2B, reaction II). In some Grampositive bacteria, Lipid II (Fig. 2A) is further decorated by an interpeptide bridge [a pentaglycine peptide in the case of $S$. aureus (11)] (Fig. 2B, reaction III) before it gets translocated across the cytoplasmic membrane to the outside, where it is incorporated into the peptidoglycan polymer through the activity of transglycosylases and transpeptidases (Fig. 2B, reaction IV). We analyzed the intracellular pool of cell-wall precursors by means of reverse high-performance liquid chromatography (HPLC) and mass spectrometry and found accumulation of the soluble molecule UDP-MurNAc-pentapeptide in plectasin-treated cells (Fig. 1D), suggesting that one of the later membrane-associated or extracellular processes may be targeted by plectasin.
We then analyzed the effect of plectasin on the membrane-bound steps of cell-wall biosynthesis in vitro. Cytoplasmic membranes with associated cell-wall biosynthesis apparatus were isolated and incubated with plectasin and radiolabeled substrates that are necessary for Lipid II formation. Using thin-layer chromatography and subsequent scintillation counting, we found the overall synthesis reaction to be strongly inhibited (Fig. 2C). For a more detailed analysis, we cloned the individual cell-wall biosynthesis genes from $S$. aureus, expressed them in Escherichia coli, and analyzed the activity of the purified enzymes in the presence of plectasin by measuring the amount of product formed. These enzymes included MraY (Fig. 2B, reaction I), MurG (Fig. 2B, reaction II), FemXAB (Fig. 2B, reaction III), and PBP2 (Fig. 2B, reaction IV). Whereas the MraY reaction was not affected
A

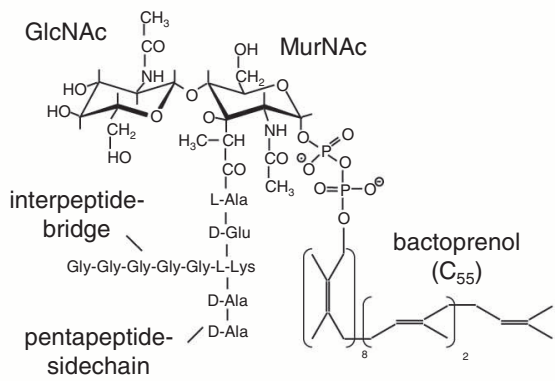

C

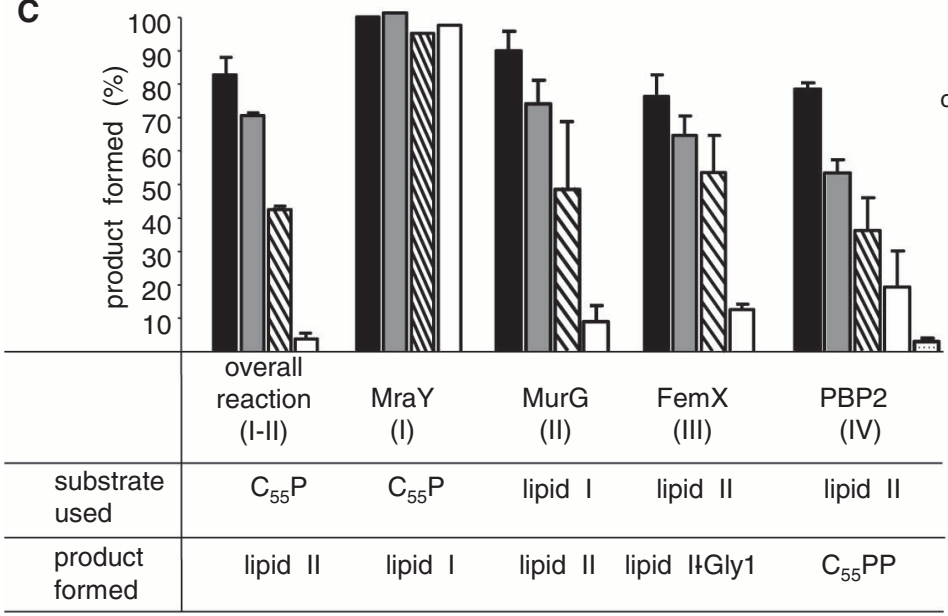

plectasin :

substrate ratio

$0.1: 1$

$0.25: 1 \quad 0.5: 1$
B

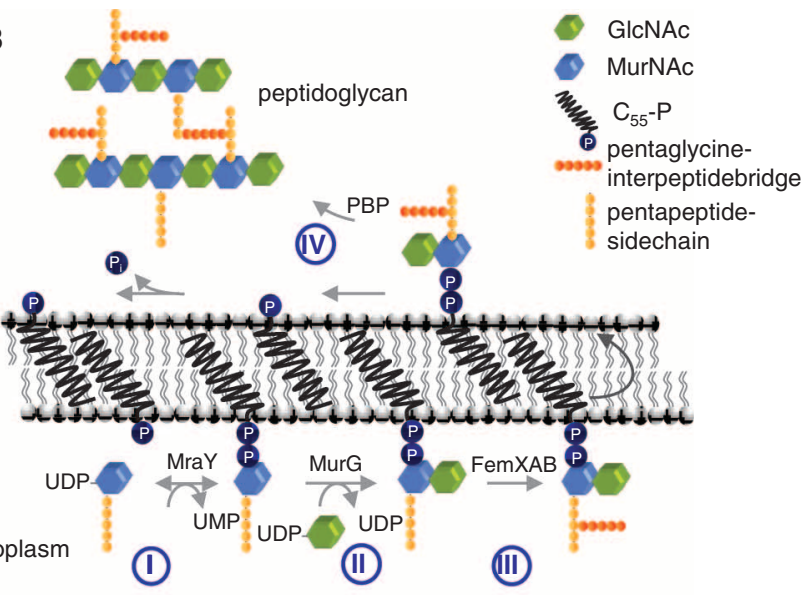

D

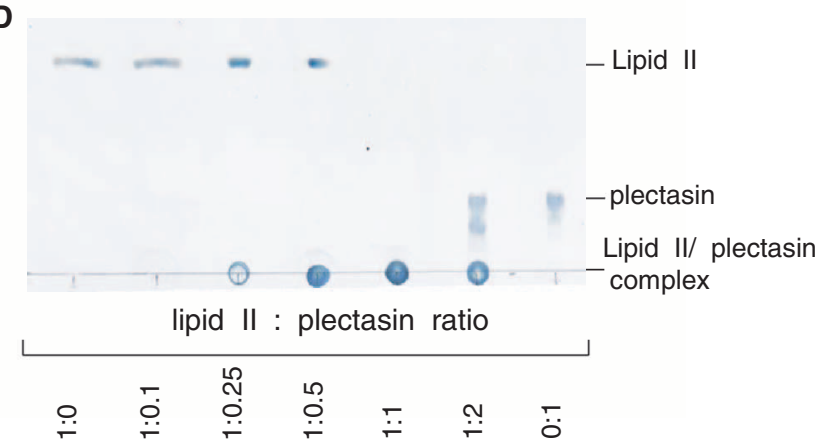

Fig. 2. Inhibition of membrane-associated cell-wall biosynthesis steps. (A) Structure of the cell-wall precursor Lipid II. (B) The membrane-bound steps of cell-wall precursor biosynthesis and bactoprenol $\left(\mathrm{C}_{55} \mathrm{P}\right)$ carrier cycling in staphylococci. Cell-wall biosynthesis starts in the cytoplasm with the formation of the soluble precursor UDP-MurNAc-pentapeptide (UDP-MurNAc-pp). This precursor is linked to the membrane carrier bactoprenolphosphate $\left(C_{55} P\right)$ by MraY yielding Lipid I (reaction I). Lipid II is formed by MurG, which adds $\mathrm{N}$-acetyl-glucosamine (GlcNAc) (reaction II). When the interpeptide bridge, which only occurs in some Gram-positive bacteria, is accomplished (reaction III), the monomeric peptidoglycan unit is translocated across the cytoplasmic membrane to the outside and incorporated into the cell wall (reaction IV). (C) Inhibition of membrane-associated steps of cell-wall biosynthesis by plectasin. In all tests, plectasin was added in molar ratios of 0.1 to 1 with respect to the amount of the appropriate lipid substrate $C_{55} \mathrm{P}$, Lipid I, or Lipid II used in the individual test system. The amount of reaction products synthesized in the absence of plectasin was taken as $100 \%$. Product analysis was done by means of TLC and subsequent scintillation counting of stained and excised productcontaining bands; radiolabeling was based on $\left.{ }^{3} \mathrm{H}\right]$-labeled $\mathrm{C}_{55} \mathrm{P}$ (for Lipid I), $\left[{ }^{14} \mathrm{C}\right]$-GlcNAc for Lipid II, and $\left[{ }^{14} \mathrm{C}\right]$-glycine for Lipid II-Gly1. Error bars represent $\pm S D$, and the experiments were repeated at least three times. Technical details on the assays and the cloning and purification of the enzymes are given in (3). (D) Estimation of the stoichiometry of plectasin:Lipid II binding. Lipid II was incubated in the presence of plectasin at the molar concentration ratios indicated. The stable complex of plectasin with the Lipid II remains at the application spot, whereas both components migrate to the sites indicated. At a molar ratio of 1:1, neither free Lipid II nor free plectasin were observed. 
by plectasin, we found the MurG, FemX, and PBP2 reactions to be inhibited in a dose-dependent fashion (Fig. 2C). For these three enzymes, Lipid I (MurG) or Lipid II (FemX and PBP2) are substrates, and significant inhibition of the reactions was only observed when plectasin was added in equimolar concentrations with respect to Lipid I or Lipid II (Fig. 2C). Thus, plectasin - similarly to glycopeptide antibiotics [such as vancomycin $(12,13)]$ and lantibiotics $(14,15)$ - may form a stoichiometric complex with the substrate rather than inhibiting the enzyme. To further validate this, we incubated either Lipid I or II with plectasin in various molar ratios and used thin-layer chromatography (TLC) to analyze the migration behavior. Free Lipid I and II as well as free peptide were found to migrate to defined positions in the chromatogram, whereas the Lipid I/II-plectasin complex remained at the start point (Fig. 2D). Free Lipid I/II and free peptide were not detectable only at an equimolar ratio, indicating the formation of a 1:1 stoichiometric complex.

We further analyzed the interaction of both Lipid I and II with plectasin using a liposome system with membranes composed of phosphatidylcholine and Lipid II [0.2 or 0.5 mole percent (mol \%)] and ${ }^{14} \mathrm{C}$-labeled plectasin. We found the maximum number of plectasin molecules that bound to liposomes to approximately match the number of Lipid II molecules available on the liposome surface (fig. S4). Using Scatchard plot analysis, we determined an equilibrium-binding constant of $1.8 \times 10^{-7} \mathrm{~mol}$ for Lipid II and $1.1 \times$ $10^{-6}$ mol for Lipid I, suggesting that the second sugar in Lipid II, the N-acetyl glucosamine, contributes to the stability of the complex.

To gain further insight into the structural nature of the plectasin/Lipid II interaction at the membrane interface, we measured chemical shift changes for ${ }^{15} \mathrm{~N}$-labeled plectasin. Heteronuclear single-quantum coherence (HSQC) nuclear magnetic resonance (NMR) spectra were measured either in solution or on binding membrane-mimicking dodecylphosphocholine (DPC) micelles (figs. S5 and S6). Fitting the binding data to a Langmuir isotherm yielded a free enthalpy of binding $\Delta \mathrm{G}=$
$-27 \pm 1 \mathrm{~kJ} / \mathrm{mol}$ (fig. S7). Backbone $\mathrm{H}^{\mathrm{N}}$ and $\mathrm{N}$ atoms of 10 residues [G6, W8, D9, A31, K32, G33, G34, F35, V36, and C37 (16)], which in the tertiary structure all locate to one end of plectasin, exhibited marked changes in chemical shifts $\left[\Delta \delta_{\text {obs }}>0.15\right.$ parts per million (ppm)] (Fig. $3 \mathrm{~A}$, residues labeled yellow), suggesting an orientation in which one end of plectasin specifically is located in the membrane interface.

To identify the residues on plectasin that bind Lipid II, we then titrated plectasin bound to DPC with Lipid II. With increasing concentrations of Lipid II, another set of NMR signals appeared and became stronger, whereas the NMR signals of apo-plectasin bound to DPC micelles became weaker until they disappeared at equimolar concentrations of plectasin and Lipid II, supporting the $1: 1$ binding stoichiometry found by means of TLC. Addition of extra plectasin to the mixture brought the signals of apo-plectasin forward again, and further addition of Lipid II to equimolarity led to the disappearance of the signals again. From a three-dimensional (3D)-HNCA spectrum, we could assign backbone $\mathrm{H}^{\mathrm{N}}, \mathrm{N}$, and $\mathrm{C}^{\alpha}$ signals of the plectasin:Lipid II:DPC complex. The strongest changes in chemical shift $\left(\Delta \delta_{\text {obs }}>0.22 \mathrm{ppm}\right)$ were obtained for amino acids F2, C4, D12, Y29, A31, G33, C37, and K38 (figs. S5 and S6). Most of these residues localize in a coherent patch in close proximity to the residues affected by binding to DPC (Fig. 3B, residues labeled magenta). A31, G33, and C37 exert chemical shift-changes both upon addition of DPC and Lipid II. To further verify this, site-saturated mutagenesis (in which a given amino acid is changed to each of the other 19 natural amino acids) was carried out at all positions in plectasin except the six cysteines. The mutant libraries were expressed in S. cerevisiae, and 400 to 600 transformants of each position tested for activity against $S$. aureus in a plate overlay assay. No amino acid substitutions at positions D12, Y29, or G33 resulted in activity against $S$. aureus, whereas only the very conservative mutations of $\mathrm{A} 31$ to $\mathrm{G}$ and $\mathrm{K} 38$ to R resulted in activity against $S$. aureus. At other amino acid positions not involved in DPC or Lipid II binding, a wide range of non-homologous amino acid substitutions gave rise to plectasin variants retaining antimicrobial activity.

To visualize the complex between Lipid II and plectasin, docking studies using the GOLD and HADDOCK programs were performed $(17,18)$. In accordance with the NMR data, evidence in favor of a primary binding site involving the interaction of the pyrophosphate moiety of Lipid II with the amide protons F2, G3, C4, and $\mathrm{C} 37$ of plectasin via hydrogen bonding was obtained (Fig. 3C). Several of the other large chemical shift changes are present in residues involved in secondary structure interactions (such as the formation of beta-sheets), which most likely undergo structural changes upon binding to the target. Taken together, these data strongly support a model in which plectasin gains affinity and specificity through binding to the solvent-exposed part of Lipid II, whereas the hydrophobic part of plectasin is located in the membrane interface. Thus, plectasin shares functional features with the lantibiotic nisin in that for both peptides the pyrophosphate moiety is most relevant for binding of Lipid II, although nisin inserts deeply into the membrane bilayer, forming pores and causing major delocalization of Lipid II $(19,20)$.

To test whether inhibition of cell-wall biosynthesis is restricted to plectasin or represents a general feature, we tested a series of defensin peptides from other fungi, mollusks, and arthropods for Lipid II binding and inhibition of the overall Lipid II synthesis and FemX reaction (fig. S8A). Two fungal defensins, oryzeasin (from Aspergillus oryzea) and eurocin (from Eurotium amstelodami), did inhibit the enzymatic reactions and bind to Lipid II in stoichiometric numbers, as did the two defensins from invertebrates, lucifensin from maggots of the blowfly Lucilia sericata and gallicin from the mussel Mytilus galloprovinciali (fig. S8, B to D). In contrast, heliomicin from the tobacco budworm Heliothis virescens, which shares the conserved cysteine pattern, did not show affinity for Lipid II and had no activity in these assays. These data clearly demonstrate that among the host defense peptides of eukaryotic
Fig. 3. NMR-based model of the plectasin/ Lipid II-complex. (A) Surface representation of plectasin with the residues showing substantial chemical shift perturbations upon binding to DPC micelles, which are indicated in yellow. (B) Surface representation of plectasin with the residues showing substantial chemical shift perturbations upon Lipid II titration, which is shown in magenta. (C) Detailed view of the pyrophosphate-binding pocket. In this proposed HADDOCK-generated model, the pyrophosphate moiety forms hydrogen bonds to F2, G3, C4, and C27, and the D- $\gamma$-glutamate of Lipid II forms a salt bridge with the $\mathrm{N}$ terminus of plectasin and the side-chain of His18.

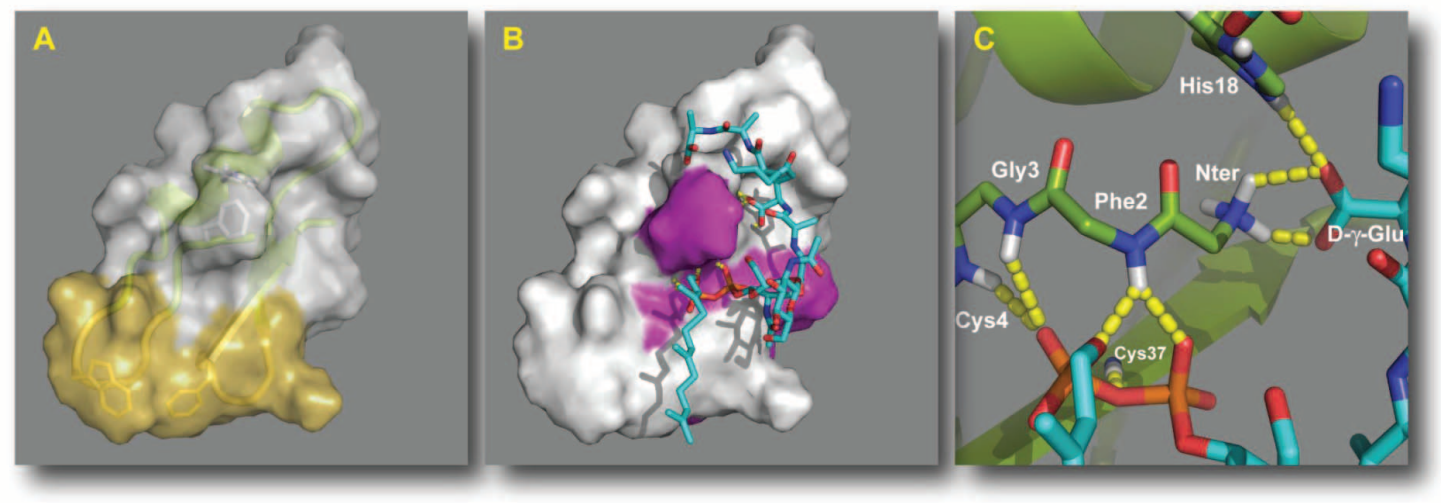

www.sciencemag.org SCIENCE VOL $328 \quad 28$ MAY 2010 
organisms, specific inhibitors of cell-wall biosynthesis can be found that directly target Lipid II, "the bacterial Achilles' heel" for antibiotic attack (21).

Vancomycin, one of the very few remaining drugs for the treatment of multi-resistant Grampositive infections, has been shown to predominantly bind the D-alanyl-D-alanine (D-ala-D-ala) part of the pentapeptide in Lipid II (Fig. 2A) (12). However, high-level vancomycin resistance has been observed in both enterococci (VRE) and staphylococci (VRSA). There is no cross-resistance between vancomycin and plectasin, and in contrast to vancomycin, plectasin is not competitively inhibited by the presence of the D-ala-D-ala ligand (fig. S9). This further demonstrates that the primary interactions to Lipid II differ between plectasin and vancomycin, and taken together, these results suggest that future development of true cross-resistance between vancomycin and plectasin is unlikely.

Plectasin and its improved derivatives such as NZ2114 possess a range of features - such as potent activity in vitro under physiological conditions and in animal models of infection, low potential for unwanted toxicities, extended serum stability and in vivo half-life, and cost-effective large-scale manufacturing - which combined with a validated microbial target make it a promising lead for further drug development.

\section{References and Notes}

1. P. H. Mygind et al., Nature 437, 975 (2005).

2. D. Andes, W. Craig, L. A. Nielsen, H. H. Kristensen, Antimicrob. Agents Chemother. 53, 3003 (2009).

3. Materials and methods are available as supporting material on Science Online.

4. M. Zasloff, Nature 415,389 (2002).

5. R. E. Hancock, H. G. Sahl, Nat. Biotechnol. 24, 1551 (2006).

6. B. Hutter et al., Antimicrob. Agents Chemother. 48, 2838 (2004).

7. F. McAleese et al., J. Bacteriol. 188, 1120 (2006).

8. M. Cao, T. Wang, R. Ye, J. D. Helmann, Mol. Microbiol. 45, 1267 (2002)

9. T. Mascher, N. G. Margulis, T. Wang, R. W. Ye, J. D. Helmann, Mol. Microbiol. 50, 1591 (2003)

10. ]. van Heijenoort, Microbiol. Mol. Biol. Rev. 71, 620 (2007).

11. T. Schneider et al., Mol. Microbiol. 53, 675 (2004).

12. P. E. Reynolds, Eur. J. Clin. Microbiol. Infect. Dis. 8, 943 (1989).

13. N. E. Allen, T. I. Nicas, FEMS Microbiol. Rev. 26, 511 (2003).

14. H. Brötz et al., Mol. Microbiol. 30, 317 (1998).

15. J. M. Willey, W. A. van der Donk, Annu. Rev. Microbiol. 61, 477 (2007)

16. Single-letter abbreviations for the amino acid residues are as follows: A, Ala; C, Cys; D, Asp; E, Glu; F, Phe; G, Gly; H, His; I, Ile; K, Lys; L, Leu; M, Met; N, Asn; P, Pro; Q, Gln; R, Arg; S, Ser; T, Thr; V, Val; W, Trp; and Y, Tyr.

17. G. Jones, P. Willett, R. C. Glen, A. R. Leach, R. Taylor, J. Mol. Biol. 267, 727 (1997).

18. C. Dominguez, R. Boelens, A. M. Bonvin, J. Am. Chem. Soc. 125, 1731 (2003)

19. S. T. Hsu et al., Nat. Struct. Mol. Biol. 11, 963 (2004)

20. H. E. Hasper et al., Science 313, 1636 (2006).
21. E. Breukink, B. de Kruijff, Nat. Rev. Drug Discov. 5, 321 (2006).

22. We thank M. Josten, A. Hansen, and M. R. Markvardsen for expert technical assistance and acknowledge the Carlsberg Research Center for use of the 800-MHz NMR spectrometer and the Obel Foundation for supporting the NMR laboratory at Aalborg University. H.G.S. acknowledges financial support by the European Union (PIAP-GA-2008-218191), the German Research Foundation (SA 292/10-2 and SA 292/13-1), the Bundesministerium für Bildung und Forschung (SkinStaph), and by the BONFOR program of the Medical Faculty, University of Bonn. A.M.].].B. acknowledges financial support from the Netherlands Organization for Scientific Research ( $\mathrm{VICl}$ grant 700.56.442). A.S.A. acknowledges financial support from the Danish Research Council for Technology and Production (274-05-0435). Novozymes AS holds a patent on plectasin (patent number W02003/044049/044049) and has filed patent applications on improved variants of plectasin. L.D.M., A.K.N., and D.S.R. hold stock options in Novozymes AS. DNA microarray data can be accessed through ArrayExpress, accession number E-MTAB-60. NMR assignment of ${ }^{1} \mathrm{H},{ }^{15} \mathrm{~N}$, and ${ }^{13} \mathrm{C}$ atoms of plectasin have been deposited in the BioMagResBank (accession number 16739).

\section{Supporting Online Material}

www.sciencemag.org/cgi/content/full/328/5982/1168/DC1

Materials and Methods

Figs. $\mathrm{S} 1$ to $\mathrm{S} 9$

Tables S1 and S2

References

8 December 2009; accepted 5 April 2010 $10.1126 /$ science. 1185723

\section{mTORC1-Mediated Cell Proliferation, But Not Cell Growth, Controlled by the $4 E-B P s$}

Ryan J. 0. Dowling, ${ }^{{ }^{*}} \dagger$ Ivan Topisirovic, ${ }^{{ }^{1 *}}$ Tommy Alain, ${ }^{1}$ Michael Bidinosti, ${ }^{1}$ Bruno D. Fonseca, ${ }^{1}$ Emmanuel Petroulakis, ${ }^{1}$ Xiaoshan Wang, ${ }^{1}$ Ola Larsson, ${ }^{1}$ Anand Selvaraj, ${ }^{2}$ Yi Liu, ${ }^{3}$ Sara C. Kozma, ${ }^{2}$ George Thomas, ${ }^{2}$ Nahum Sonenberg ${ }^{1} \ddagger$

The mammalian target of rapamycin complex 1 (mTORC1) integrates mitogen and nutrient signals to control cell proliferation and cell size. Hence, mTORC1 is implicated in a large number of human diseases - including diabetes, obesity, heart disease, and cancer-that are characterized by aberrant cell growth and proliferation. Although eukaryotic translation initiation factor 4E-binding proteins (4E-BPs) are critical mediators of mTORC1 function, their precise contribution to mTORC1 signaling and the mechanisms by which they mediate mTORC1 function have remained unclear. We inhibited the mTORC1 pathway in cells lacking 4E-BPs and analyzed the effects on cell size, cell proliferation, and cell cycle progression. Although the 4E-BPs had no effect on cell size, they inhibited cell proliferation by selectively inhibiting the translation of messenger RNAs that encode proliferation-promoting proteins and proteins involved in cell cycle progression. Thus, control of cell size and cell cycle progression appear to be independent in mammalian cells, whereas in lower eukaryotes, 4E-BPs influence both cell growth and proliferation.

$\mathrm{T}$ he mammalian target of rapamycin complex 1 (mTORC1) controls growth (increase in cell mass) and proliferation (increase in cell number) by modulating mRNA translation through phosphorylation of the eukaryotic translation initiation factor 4E (eIF4E)binding proteins (4E-BP1, 2, and 3) and the ribosomal protein S6 kinases (S6K1 and 2) $(1,2)$. 4E-BPs regulate the translation of a subset of
mRNAs by competing with eIF4G for binding to eIF4E, thus preventing the assembly of the eIF4F complex, whereas the S6Ks control the phosphorylation status of a number of translational components (1-3). Rapamycin has been an important tool in understanding mTORC1 signaling; however, it inefficiently and transiently inhibits 4E-BP phosphorylation (4) (fig. S1A). Moreover, we found that rapamycin inhibited proliferation and $G_{1} / S$ cell cycle progression of wild-type and 4E-BP double-knockout (DKO) mouse embryonic fibroblasts (MEFs) to the same extent, which suggests that its effects are not mediated by 4E-BPs (fig. S1, B to D). To directly address the role of 4E-BPs in mTORC1 signaling, we depleted raptor, a component of mTORC1 required for substrate binding (5), in these MEFs. 4E-BP DKO MEFs lack all three 4E-BPs, as they do not express 4E-BP3 (fig. S2A). Depletion of raptor diminished the phosphorylation of $4 \mathrm{E}-\mathrm{BP} 1$ at all mTORsensitive sites in wild-type MEFs, and inhibited mTORC1 signaling to the same extent in wildtype and 4E-BP DKO MEFs, as illustrated by reduced phosphorylation of S6Ks and its substrates (ribosomal protein S6 and eIF4B) and increased abundance of programmed cell death protein 4 (PDCD4) (Fig. 1A). Wild-type MEFs in which raptor was depleted proliferated more slowly than control cells, whereas raptor-depleted 4E-BP DKO MEFs proliferated at a rate indistinguishable from that of control cells (Fig. 1B). Similarly, in human embryonic kidney (HEK)

${ }^{1}$ Department of Biochemistry and Goodman Cancer Research Centre, McGill University, Montreal, Quebec H3A 1A3, Canada. ${ }^{2}$ Department of Cancer and Cell Biology, Metabolic Diseases Institute, University of Cincinnati, Cincinnati, $\mathrm{OH}$ 45237, USA. ${ }^{3}$ Intellikine, La Jolla, CA 92037, USA.

*These authors contributed equally to this work. †Present address: Division of Signalling Biology, Ontario Cancer Institute, University Health Network, Toronto, Ontario M5G 2M9, Canada.

‡To whom correspondence should be addressed. E-mail: nahum.sonenberg@mcgill.ca 


\section{Science}

\section{Plectasin, a Fungal Defensin, Targets the Bacterial Cell Wall Precursor Lipid II}

Tanja Schneider, Thomas Kruse, Reinhard Wimmer, Imke Wiedemann, Vera Sass, Ulrike Pag, Andrea Jansen, Allan K. Nielsen, Per H. Mygind, Dorotea S. Raventós, Søren Neve, Birthe Ravn, Alexandre M. J. J. Bonvin, Leonardo De Maria, Anders S. Andersen, Lora K. Gammelgaard, Hans-Georg Sahl and Hans-Henrik Kristensen

Science 328 (5982), 1168-1172.

DOI: $10.1126 /$ science.1185723

\section{Defining Defensins' Mode of Action}

Defensins are antimicrobial host defense peptides that play a role in innate immunity. Many such peptides act by disrupting the bacterial membrane; however, Schneider et al. (p. 1168) now show that the fungal defensin, plecstasin, targets cell wall biosynthesis. Biochemical studies identified Lipid II as the cellular target of plecstasin and the residues involved in complex formation were identified using NMR spectroscopy and computational modeling. Initial studies identified two defensins from invertebrates that also target Lipid II. Plecstasin is active against some drug-resistant Gram-positive bacteria, and its action against a validated target makes it a promising lead for further drug development.

ARTICLE TOOLS

SUPPLEMENTARY MATERIALS

REFERENCES

PERMISSIONS http://science.sciencemag.org/content/328/5982/1168

http://science.sciencemag.org/content/suppl/2010/05/24/328.5982.1168.DC1

This article cites 19 articles, 5 of which you can access for free http://science.sciencemag.org/content/328/5982/1168\#BIBL

http://www.sciencemag.org/help/reprints-and-permissions

Use of this article is subject to the Terms of Service

Science (print ISSN 0036-8075; online ISSN 1095-9203) is published by the American Association for the Advancement of Science, 1200 New York Avenue NW, Washington, DC 20005. The title Science is a registered trademark of AAAS.

Copyright @ 2010, American Association for the Advancement of Science 